\title{
Influence of strain rate on the mechanical behaviour in tension of bovine cortical bone
}

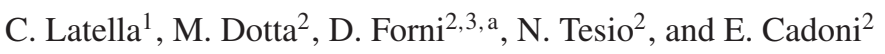 \\ ${ }^{1}$ University of Genova, Italy \\ ${ }^{2}$ DynaMat Lab, University of Applied Sciences of Southern Switzerland, 6952 Canobbio, Switzerland \\ ${ }^{3}$ Department of Structural, Geotechnical and Building Engineering, Politecnico di Torino, Torino, Italy
}

\begin{abstract}
The mechanical behaviour of bones when subjected to tension loading in a wide range of strain-rates is fundamental to develop protection systems. The paper presents the preliminary tests on the tensile behaviour of bovine cortical bone at medium and high strain rates. Two special apparatus, both installed at the DynaMat Laboratory of the University of Applied Sciences of Southern Switzerland, a Hydro-Pneumatic Machine and a Modified Hopkinson Bar respectively for medium and high strain-rate tests have been used. Flat shape specimens (having $10 \mathrm{~mm}$ of gauge length, $5 \mathrm{~mm}$ width and $3 \mathrm{~mm}$ thickness) have been obtained from 15 bovine femurs with the same age. The paper describes the preparation techniques of the samples and the experimental results obtained. The bovine cortical bone shown a quite important strain rate dependency.
\end{abstract}

\section{Introduction}

In human and other mammal bodies, bone is generally classified into two different types, trabecular and cortical bone, known also as cancellous or spongy bone and compact bone respectively.

On the basis of the porosity and the unit microstructure classification, the cortical bone, as is known, is much denser, harder and stiffer than the cancellous bone. This type of bone, that is the major component of the human body, makes up nearly $80 \%$ of skeletal mass and is of vital importance to weight bearing and as a protective structure because of its high bending and torsional resistance.

Even if the mechanical properties measurement is a very difficult task, their quantification can be useful to study and develop theories concerning fracture mechanism that can predict failures.

For these reasons this material has been the subject of mechanical investigation for many years. In numerous studies the rate dependency nature of its behaviour has been acknowledged.

For example Pitioux [1] compared the bone failure under two different loading rates, developing a statistical model. Ferreira [2], by using a traditional SHPB, investigated the compressive mechanical properties in static and dynamic conditions of both trabecular and cortical bones. Other authors, [3], conducted a preliminary and fracture toughness test on dry and wet bovine bones under quasi-static and dynamic loading conditions. A viscoelastic, viscoplastic model of the cortical bone valid in a wide range of strain rates was proposed by Johnson [4] by fitting different sets of stressstrain measurement from the available literature data.

\footnotetext{
${ }^{a}$ Corresponding author: daniele.forni@supsi.ch
}

Hansen [5], investigated the tensile and compressive properties of human cortical bone at different strain rates.

Another consideration concerns the fracture behaviour. It is widely reported [6] that the bone is strain rate sensitive while increasing the loading rate. This behaviour is relevant to cortical bone response in both tension [7] and compression [8-10].

The purpose of this paper is to present the preliminary tests on the tensile behaviour of bovine cortical bone in a wide range of strain rates $\left(10^{-3} \div 100 \mathrm{~s}^{-1}\right)$. This could be helpful for the assessment of finite element analysis in order to improve the development of advantageous body protection systems.

\section{Experimental program}

Tensile tests at three different strain rates $\left[10^{-3}, 3,100 \mathrm{~s}^{-1}\right]$ were performed at room temperature on 15 flat dogbone samples (Fig. 1) obtained from bovine cortical bone femurs. All the specimens are stored in a frozen conditions, except during the preparation and the test. In order to reduce conditioning problems connected to the post-mortem ageing (PMA) all dynamic tests were performed during the same day. Only static tests were performed few days later.

\subsection{Preparation techniques}

The cortical femur bones were acquired in the Genova abattoir from adult bovines of nearly the same age. The specimens were extracted so that their axis was longitudinal-oriented with respect to the whole femur bone. The articulate ends (epiphysis) were removed in order to obtain only the central part (diaphysis). The derived pieces were split in a half for a more useful sample realisation and secondly, these half-pieces were 


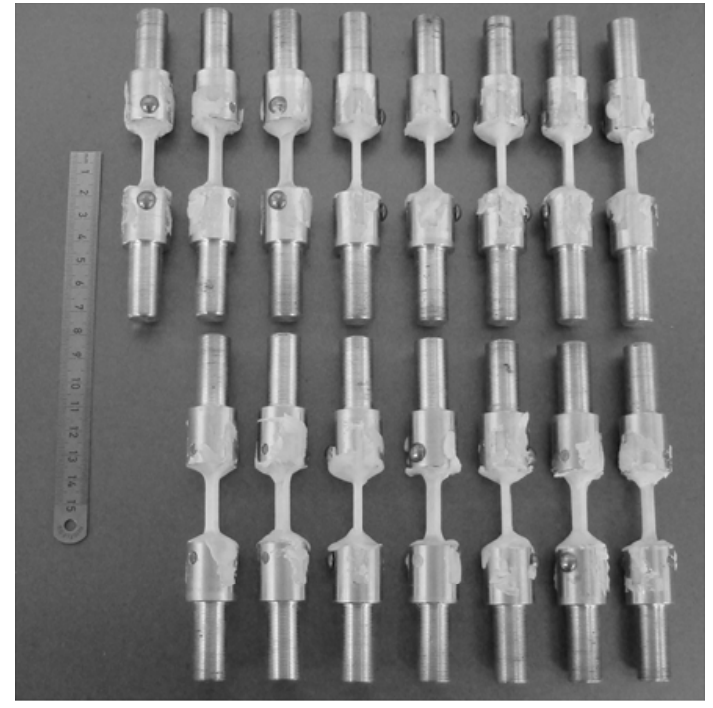

Figure 1. Samples ready for tensile tests.

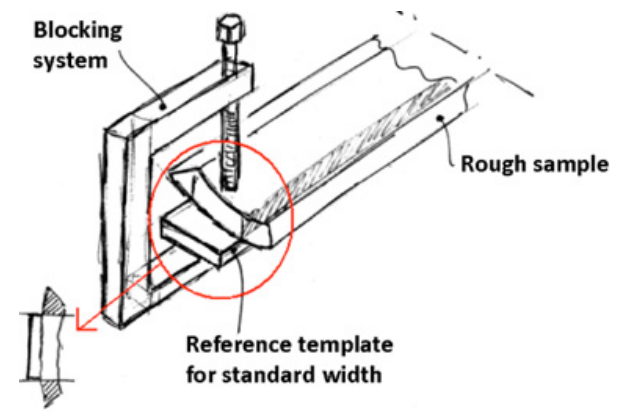

Figure 2. Sample's preparation sketch.

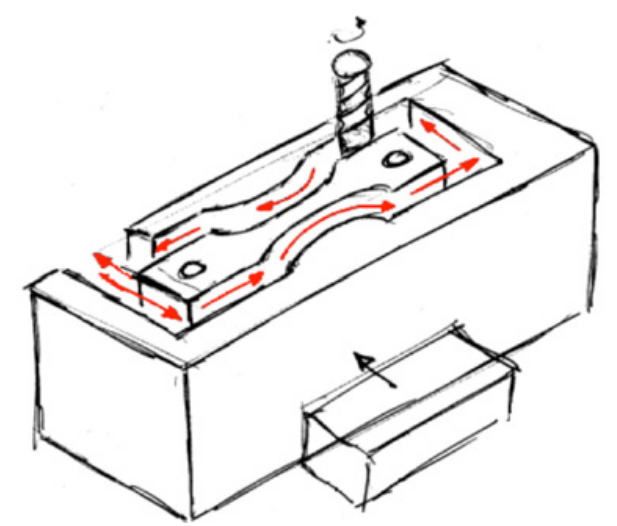

Figure 3. Sample's preparation sketch.

machined (Fig. 2) with the purpose of obtaining equal thin specimens. Then, by means of a numerical controlled machine the requested sample geometry was obtained (Fig. 3).

Lastly, with a sterile radiopaque acrylic cement for orthopaedic surgery (Biocemium I) the samples were glued and then immediately joined to the supporting holders, previously designed for the connection with the testing machines.

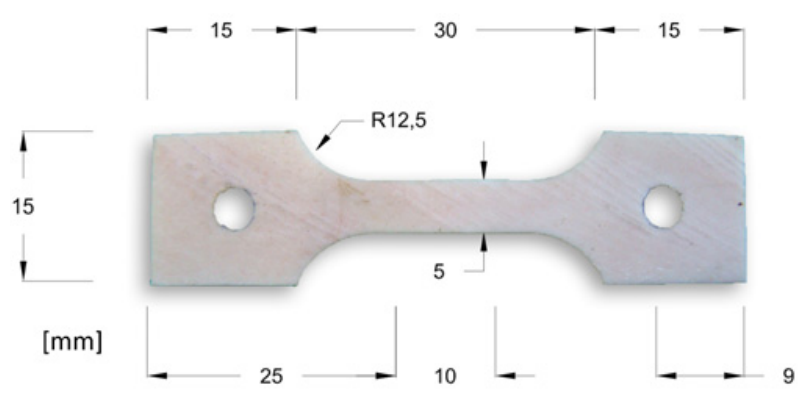

Figure 4. Sample's geometry.

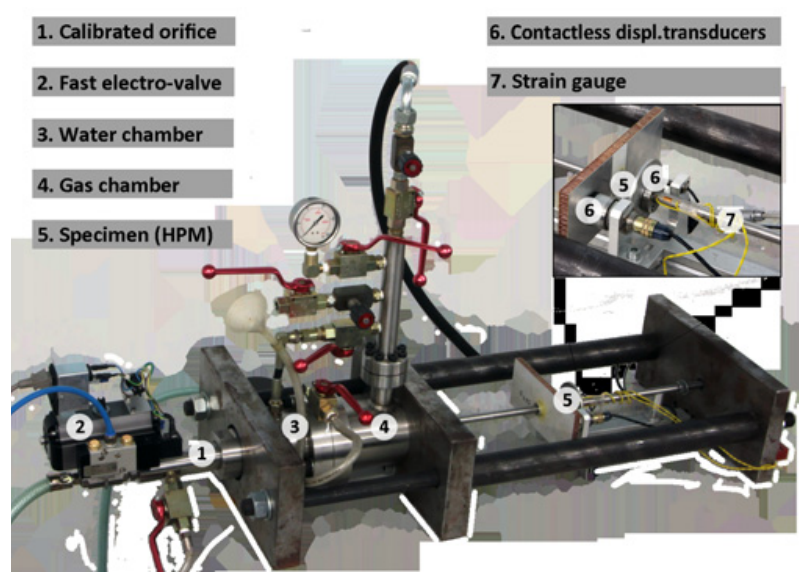

Figure 5. Hydro-pneumatic machine used in this research.

\subsection{Experimental set-ups}

Different experimental set-ups for tensile testing at several strain-rates have been used. All tests were performed at room temperature, and the target strain-rates were set at the following three levels: $10^{-3}, 3$ and $100 \mathrm{~s}^{-1}$.

\subsubsection{Quasi-static tests}

Quasi-static tests have been performed by means of a universal electromechanical testing machine Schenck $F C-400$ installed at the University of Genova.

\subsubsection{Intermediate strain rate tests}

Intermediate strain-rate behaviour in tension has been investigated by means of a hydro-pneumatic machine (HPM), whose scheme is reported in Fig. 5. The functioning of the machine is well described here $[11,12]$ and is briefly reported as follow.

Firstly, the water is filled in the upper tank-chamber (3) and the gas is filled in the lower tank-chamber (4). At this moment, equal pressure is established in both chambers so that the forces acting on the two piston faces are in equilibrium. Secondly, the specimen (5) is fixed to both the piston shaft and the elastic bar instrumented with a strain gauge (7). Lastly, by activating a fast electro-valve (2) closing the water chamber, the force exerted by the gas pressure on one face of the piston prevails, accelerating the piston, which simultaneously loads the specimen and pushes water to flow out through the calibrated orifice (1) at a constant speed, with the result of imposing a strain to the specimen with a constant strain-rate. The stress 


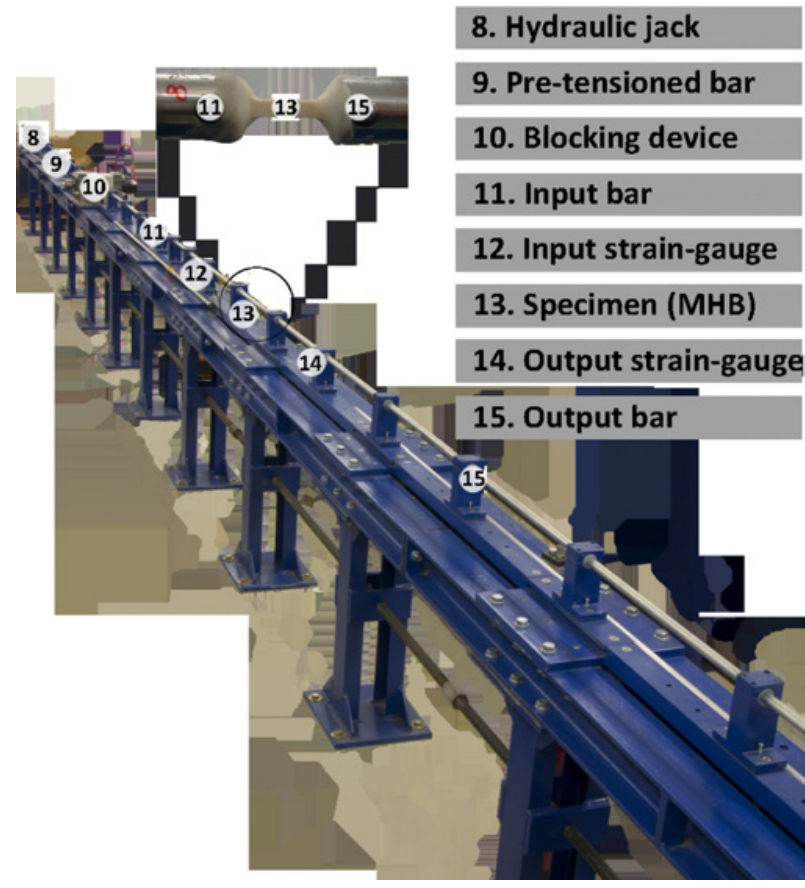

Figure 6. Split Hopkinson Tensile bar used in this research.

measurements were obtained by the strain gauge of the elastic bar connected to the specimen while the strain measurements were obtained by means of two contactless displacement transducers (6) placed at the ends of the specimen.

\subsubsection{High strain rate tests}

The high strain-rate tests in tension were performed using a Split Hopkinson Tensile Bar (SHTB) [13], that consists of two circular aluminium bars, called input and output bars and having a length of $3 \mathrm{~m}$ and $6 \mathrm{~m}$ respectively, with a diameter of $20 \mathrm{~mm}$. The specimen is screwed between these bars (Fig. 6). The input bar is connected with an high strength steel pre-tensioned bar, $6 \mathrm{~m}$ in length, used as pulse generator with a diameter of $12 \mathrm{~mm}$ in order to obtain the same acoustical impedance of the input bar so that the pulse reflection, due to the interface, is avoided.

With reference of Fig. 6, a test with the SHTB is performed as follows: firstly, the hydraulic actuator (8) of maximum loading capacity of $600 \mathrm{kN}$ is pulling the pretension high strength steel bar (9). The pretension stored in this bar is resisted by the blocking device (10). Secondly, the rupture of a fragile bolt in the blocking device, gives rise to a tensile mechanical pulse of $2,4 \mathrm{~ms}$ with linear loading rate during the rise time, propagating along the input (11) and the output (15) bars bringing to fracture the specimen (13).

As well as in the classical Hopkinson bar apparatus, the pulse propagates along the input bar with the elastic wave velocity equal to $C_{0}$ and constant shape. When the incident pulse $\left(\epsilon_{I}\right)$ reaches the bone sample, it is partially reflected by it $\left(\epsilon_{R}\right)$. On the other hand, the portion of the pulse that is not reflected, passes through the specimen $\left(\epsilon_{T}\right)$ and propagates into the output bar.

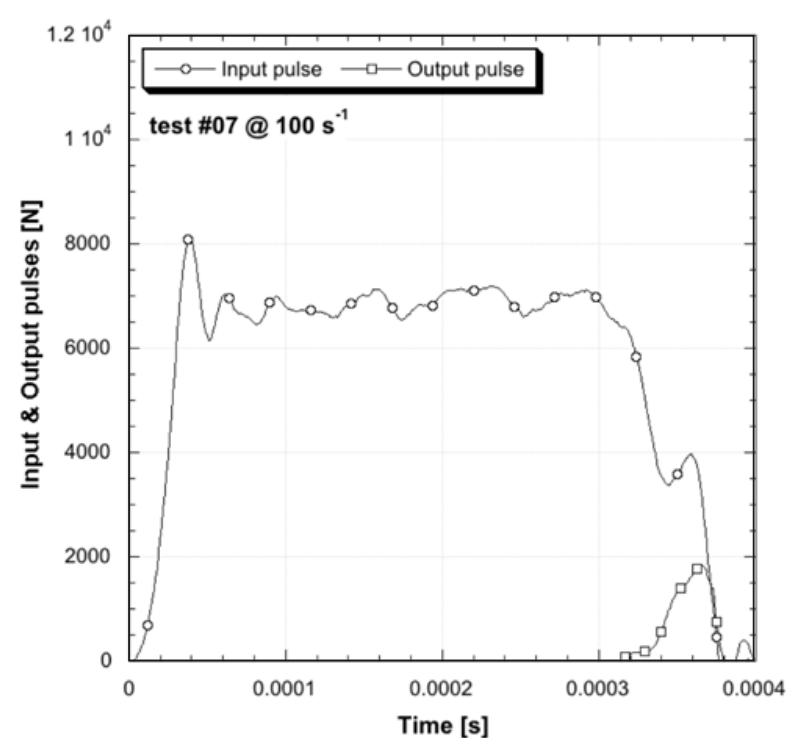

Figure 7. Input and output pulses during an high strain rate test.

The clean resolution (Fig. 7) of the incident, reflected and transmitted pulses, the sharp rise time of the incident pulse, as well as the almost constant amplitude of the incident pulse have been proved in past researches [11-13].

Thereafter, the stress, the strain as well as the strain rate in the sample can be derived from the following equations:

$$
\begin{gathered}
\sigma(t)=E_{0} \cdot \frac{A_{0}}{A_{s}} \cdot \epsilon_{T}(t) \\
\epsilon(t)=-\frac{2 \cdot C_{0}}{L_{s}} \int_{0}^{t} \epsilon_{R}(t) d t \\
\dot{\epsilon}(t)=-\frac{2 \cdot C_{0}}{L_{s}} \epsilon_{R}(t) .
\end{gathered}
$$

Where, $L_{S}$ is the specimen length, $A_{S}$ is the specimen cross-section area, $A_{0}$ is the output bar cross-section area, $E_{0}$ is the bar elastic modulus and $t$ is the time.

Using the above mentioned equations, the stress and the strain-rate versus time are plotted in Fig. 8. Due to the brittle behaviour of the bone, the conventional strain rate has been adopted as the value in correspondence of the maximum tensile stress.

\section{Results and discussion}

Tensile tests on flat dog-bone samples obtained from bovine cortical bone femurs were performed at room temperature and at three different strain rates: $10^{-3}, 3$, $100 \mathrm{~s}^{-1}$

Literature data report that usually high loading rates lead to an increase of yield and pre-yield properties as well as the elastic modulus [5]. On the other hand, postyield properties are less consistent, and both increases and decreases in strength are reported [1].

Our experimental data are depicted in Fig. 9 and Fig. 10 for tests performed at medium and at high strain rate respectively. With the purpose of pointing out 


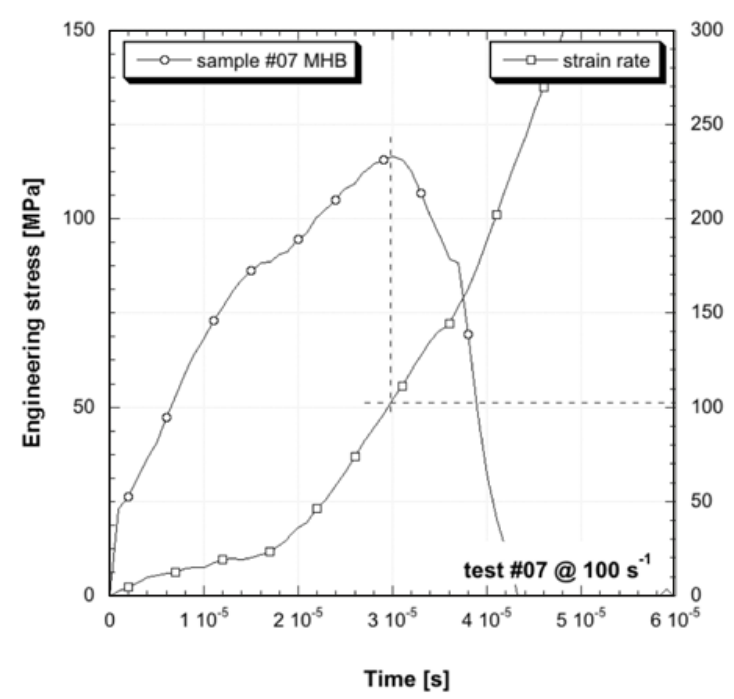

Figure 8. Stress and strain-rate versus time.

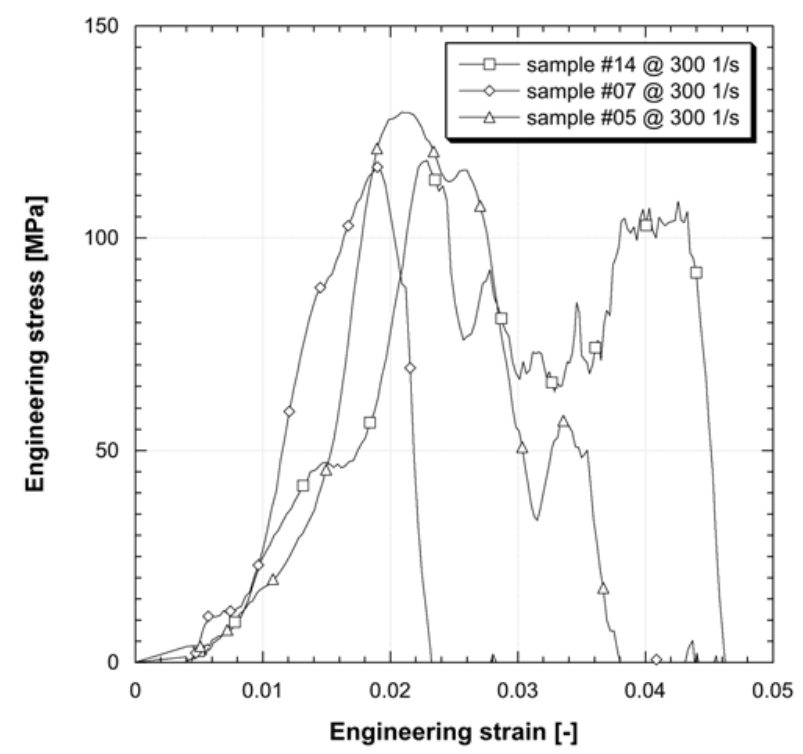

Figure 9. Comparison plot of high strain rate tests.

the strain rate sensitivity of the bovine cortical bone, a comparison between static and dynamic experimental data has been performed (Fig. 11).

In static condition the maximum engineering stress is $69.33 \pm 1.46 \mathrm{MPa}$, while at high strain rate this value become $121.50 \pm 7.04 \mathrm{MPa}$ and at intermediate strain rate is $46.65 \pm 11.81 \mathrm{MPa}$. The elastic modulus obtained in quasi-static condition was about $8.8 \mathrm{GPa}$, while at high strain rate was about $14.9 \mathrm{GPa}$. It has not been possible to estimate the elastic modulus at medium strain rate because of problems connected to the displacement measurement during these tests.

With the exception of intermediate strain rate data, whose results show a marked sensitivity to strain rate. Tests performed at $100 \mathrm{~s}^{-1}$ highlight an increase of elastic modulus, maximum stress, as well as the corresponding deformation.

As far as we know, not many researches are focused on the direct tensile behaviour of bone at high strain

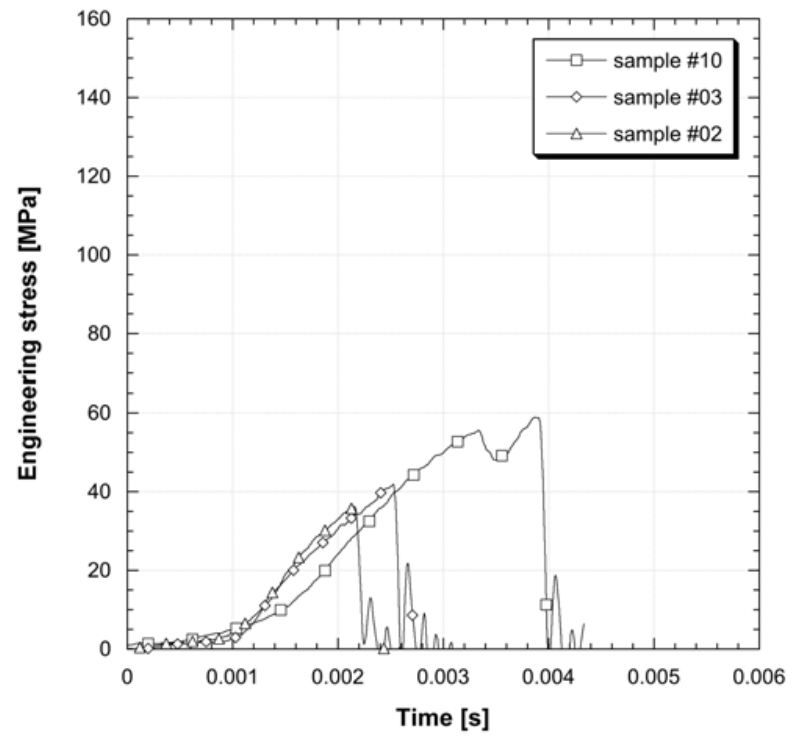

Figure 10. Comparison plot of intermediate strain rate tests.

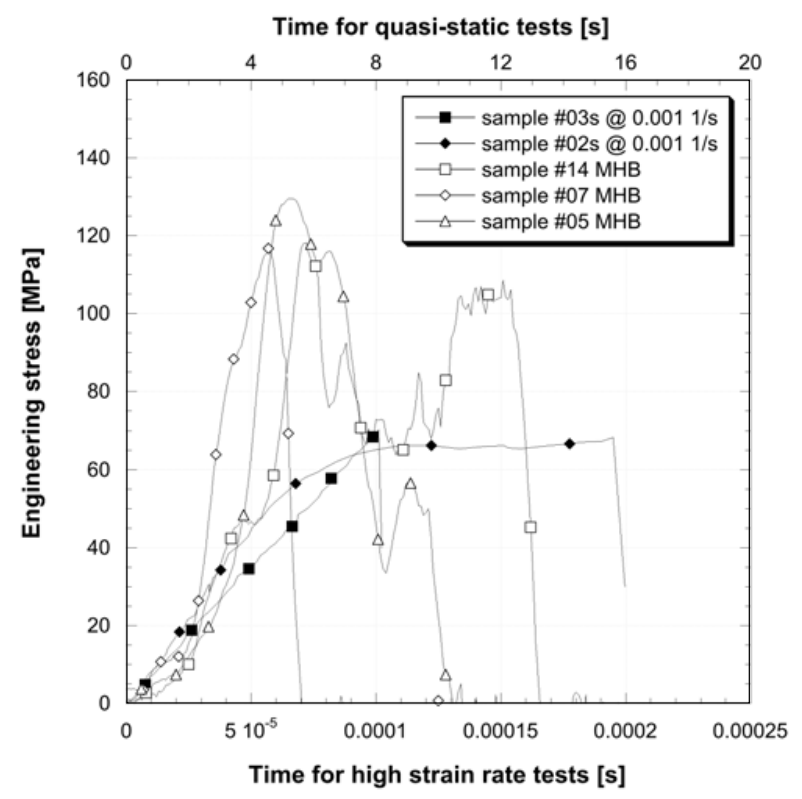

Figure 11. Different behaviour of quasi-static and high strain rate tests.

rate. Some authors [1] performed direct tensile tests at different strain rates, finding an increase of elastic modulus $11.3 \div 17.5 \mathrm{GPa}$ to $10 \div 21.7 \mathrm{GPa}$ and a decrease of both maximum stress $105 \div 193 \mathrm{MPa}$ to $33 \div 50 \mathrm{MPa}$ and strain at failure $0.93 \div 1.8 \%$ to $0.23 \div 0.44 \%$. These results are only partially in accordance with our high strain rate tests, but seems to be in conformity with the intermediate strain rate results.

Wood [14], employing an hydraulic testing machine performed tensile tests at different strain rates $\left(0.005 \div 150 \mathrm{~s}^{-1}\right)$ on more than 120 specimens obtained from human cranial bone. A regression line for elastic modulus data is given in function of the strain rate. In this research is reported that statistical analysis of the data reveals no important differences in the modulus of elasticity, the breaking stress, the breaking strain, or the 


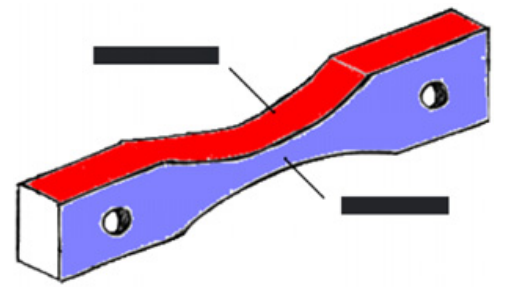

Figure 12. Sample's geometry.

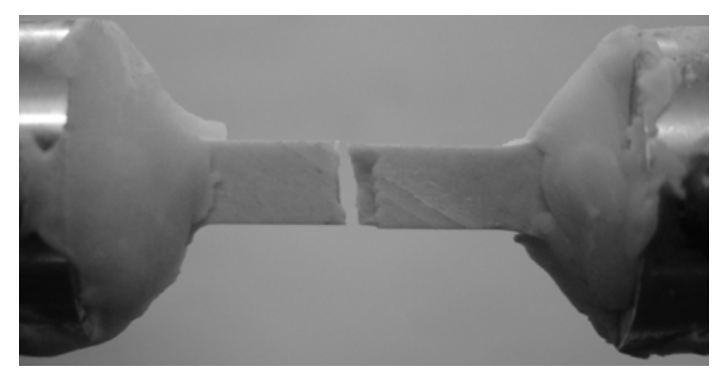

Figure 13. Sample's failure after an high strain rate test.

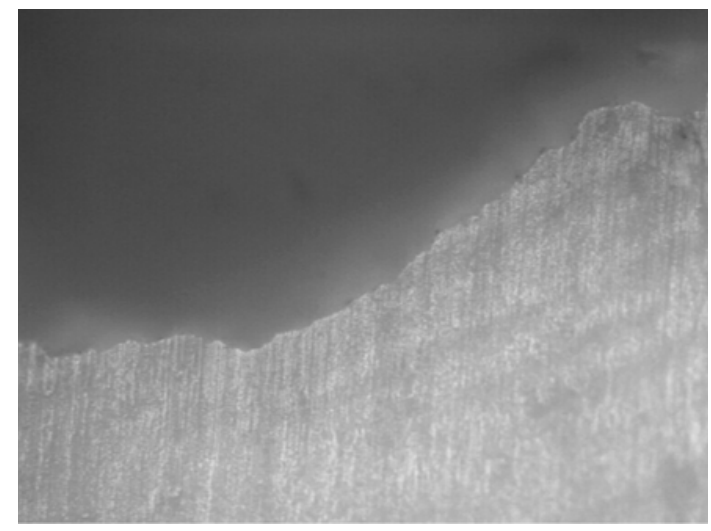

Figure 14. Dark streak lines.

energy absorbed to failure when compared according to type of bone, side of body or age of the individual. For that reason this relationship has been applied to our data. For strain rates ranging from $10^{-3} \mathrm{~s}^{-1}$ to $100 \mathrm{~s}^{-1}$ gives 10.2 $\mathrm{GPa}$ and $19.8 \mathrm{GPa}$ respectively, in good agreement with our results.

Crowninshield [7] conducted a study on bovine cortical bone in tension $\left(10^{-3} \div 250 \mathrm{~s}^{-1}\right)$ finding an increase of elastic modulus with strain rate from 10 to $12.5 \mathrm{GPa}$, that slightly underestimate our results.

With the purpose of both a better interpretation of our results and an evaluation of possible influence of samples preparation, a post-mortem analysis with an optical microscope has been performed. This analysis highlighted that the front side of the sample presents more flaws with respect to the lateral side. This could be due to the preparation process. Even though these imperfections, the most interesting aspect has been observed in the lateral side, where the direction of the fracture seems to be correlated with dark streak lines (Fig. 14). The nature of these bands should be connected with the damage morphology development during the bone fracture. As a confirmation of our hypothesis, this diffuse damage was pointed out and discussed in previous researches $[15,16]$.

\section{Conclusions}

Although intermediate strain rate data shows a negative sensitivity to strain rate, tests at $100 \mathrm{~s}^{-1}$ highlight an increase of elastic modulus, maximum stress as well as the corresponding deformation. With reference of comparable researches, it has been possible to point out that our results are in a satisfactory agreement with previously published data.

With the purpose of obtaining samples with a strict geometry useful for the mechanical characterisation, the most difficult task of this research has been the preparation of the samples. For that reason only few samples were satisfactory well prepared and ready for mechanical tests. Even if no more than 15 samples were prepared (Fig. 1), only 3 tests at each strain rate have been considered in the analysis. Future development will be focused on the improvement of samples preparation process, as well as a in-depth understanding with regards to the damage morphology development during the bone fracture.

\section{References}

[1] M. Pithioux, D. Subit and P. Chabrand, Med. Eng. Phys., 26, 647-567 (2004)

[2] F. Ferreira, M.A. Vaz and J.A. Simoes, Mater. Charact., 57, 71-79 (2006)

[3] R.R. Adharapurapu, F. Jiang and K.S. Vecchio, Mat. Sci. Eng. C, 26, 1325-1332 (2006)

[4] T.P.M. Johnson, S. Socrate and M.C. Boyce, Acta Biomater., 6 (2010)

[5] U. Hansen, P. Zioupos, R. Simpson, J.D. Currey and D. Hynd, J. Biomech. Eng. 130 (2008)

[6] A. van der Westhuizen, Ph.D. Thesis (2008)

[7] R.D. Crowninshield and M.H. Pope, Ann. Biomed. Eng., 2, 217-225 (1974)

[8] J.H. McElhaney, J. Appl. Physiol, 21, 1231-1236 (1966)

[9] A. Bekker, T.J. Cloete, A. Chinsamy-Turan, G.N. Nurick and S. Kok, Mat. Sci. Eng. C, 46, 443-449 (2015)

[10] M. Prot, D. Saletti, S. Pattofatto, V. Bousson and S. Laporte, J. Biomech., 48, 498-503 (2015)

[11] E. Cadoni, M. Dotta, D. Forni, and P. SpŁtig, J. Nucl. Mater., 414, 360-366 (2011)

[12] E. Cadoni, M. Dotta, D. Forni, N. Tesio and C. Albertini, Mater. Design, 49, 657-666 (2013)

[13] E. Cadoni, L. Fenu and D. Forni, Constr. Build. Mat., 35, 399-407 (2012)

[14] J.L. Wood, J. Biomech., 4, 1-12 (1971)

[15] T. Diab and D. Vashishth, Bone, 37, 69-102 (2005)

[16] D.B. Burr, C.H. Turner, P. Naick, M.R. Forwood, W. Ambrosius, M.S. Hasan and R. Pidaparti, J. Biomech., 31, 337-345 (1998) 